\title{
Rubidium 'whiskers' in a vapour cell
}

To the Editor - For many years, we have been using glass alkali-metal vapour cells with inner walls coated with paraffin. Highquality paraffin coatings ${ }^{1,2}$ have the 'magical' property that polarized alkali atoms may bounce between the cell walls several thousand times before they depolarize, which has led to widespread application of such cells anywhere where long-lived atomic polarization is desired, for example, in optical atomic magnetometers ${ }^{3}$.

Although condensation of the alkali metal on the paraffin-coated walls is usually carefully avoided, and the excess metal is located in the cell 'stem' (see Fig. 1a), in some of the cells, we have observed needle-like metal crystals - shown in Fig. 1 - that have grown from the paraffin coating and have reached the length of up to $2-3 \mathrm{~mm}$ in about two months (our coating procedure is described in ref. 4). The crystals are all of regular square crosssection, approximately $30 \mu \mathrm{m}$ on the side, terminating on a sharp-vertex pyramid at the ends of the needle.

The observed rate of growth indicates that an atom from the vapour phase that hits the crystal at the prismatic part of the needle near the vertex has sticking probability of order unity, or that atoms adsorbed in a 'wrong' place on the crystal somehow migrate towards the end of the needle. Most of the whiskers are perpendicular to the cell wall, but occasionally we find needles that grow at an angle.

A quick e-mail poll of the workers who have studied paraffin-coated cells has revealed that, although most of them have not seen alkali-metal whiskers, the paraffin-coating pioneer, Hugh Robinson (now at NIST, Boulder), in fact, first observed their formation some 20 years ago, and was even able to stimulate their formation by providing a 'cold spot' on the surface of the cell. Unfortunately, Robinson's results remain unpublished.

As it turns out, rubidium is a member of a large 'family' of metals and other materials that tend to grow whiskers ${ }^{5}$. In fact, tin, zinc and gold whiskers represent a significant problem for the electronics industry, as they can lead to electrical shorts and failure of electronic equipment. NASA

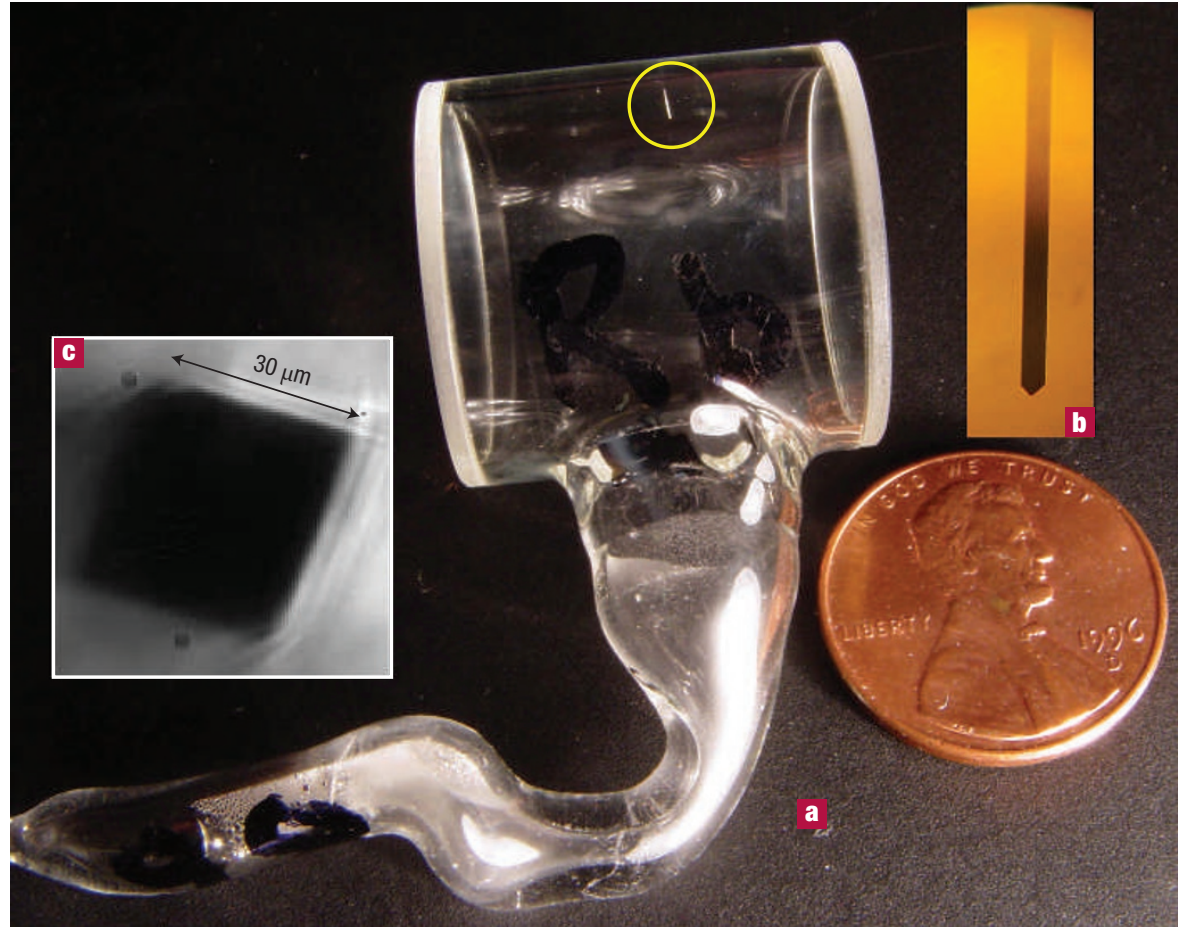

Figure 1 Rubidium whiskers. a, View of a paraffin-coated vapour cell with one of the rubidium whiskers clearly seen (outlined with a yellow circle). b, A microscope photograph of the side view of the whisker. c, Microscope photograph of the base of the whisker as seen from the coating side. (We thank A. Cingoz and D. English for help with photography.)

maintains an informative web site ${ }^{6}$ devoted to metal whiskers, however, alkali metals have not as yet been featured. There are also reports of bismuth whiskers growing in a heat-pipe vapour cell (M. Zolotorev, personal communication).

Alkali-metal needles may also present certain problems, for example, in the work that requires application of high-voltage electric field to coated cells ${ }^{7}$. However, they might also prove useful as a way to manufacture alkali-metal wires, for which one interesting application is loading a dense cryogenic buffer gas with the alkali metal by laser ablation of the end of a micrometre-sized wire ${ }^{8}$.

M. V. Balabas ${ }^{1}$, A. O. Sushkov ${ }^{2}$ and D. Budker ${ }^{3 *}$ ${ }^{1}$ S. I. Vavilov State Optical Institute, St. Petersburg 199034, Russia.
${ }^{2}$ Department of Physics, University of California, Berkeley, California 94720-7300, USA.

${ }^{3}$ Department of Physics, University of California, Berkeley and Nuclear Science Division, Lawrence Berkeley National Laboratory, Berkeley, California 94720, USA.

*e-mail:budker@berkeley.edu

\section{References}

1. Robinson, H., Ensberg, E. \& Dehmelt, H. Bull. Am. Phys. Soc. 3, 9 (1958).

2. Bouchiat, M. A. \& Brossel, J. Phys. Rev. 147, 41-54 (1966).

3. Budker, D. \& Romalis, M. V. Preprint at <http://arxiv.org/ physics/0611246> (2006).

4. Alexandrov, E. B. et al. Phys. Rev. A 66, 042903 (2002).

5. Givargizov, E. I. Highly Anisotropic Crystals (D. Reidel/Terra Scientific, 1987).

6. http://nepp.nasa.gov/whisker/

7. Budker, D., Kimball, D. F., Rochester, S. M. \& Yashchuk, V. V. Phys. Rev. A 65, 033401 (2002).

8. Sushkov, A. O. Studies of Magneto- and Electro-optical Effects at Cryogenic Temperatures Thesis Univ. California Berkeley (2006). 